\title{
Automated identification of pneumonia in chest radiograph reports in critically ill patients
}

\author{
Vincent Liu ${ }^{1,2^{*}}$, Mark P Clark ${ }^{3}$, Mark Mendoza ${ }^{2}$, Ramin Saket ${ }^{2}$, Marla N Gardner ${ }^{1}$, Benjamin J Turk \\ and Gabriel J Escobar ${ }^{1,4}$
}

\begin{abstract}
Background: Prior studies demonstrate the suitability of natural language processing (NLP) for identifying pneumonia in chest radiograph (CXR) reports, however, few evaluate this approach in intensive care unit (ICU) patients.

Methods: From a total of 194,615 ICU reports, we empirically developed a lexicon to categorize pneumonia-relevant terms and uncertainty profiles. We encoded lexicon items into unique queries within an NLP software application and designed an algorithm to assign automated interpretations ('positive', 'possible', or 'negative') based on each report's query profile. We evaluated algorithm performance in a sample of 2,466 CXR reports interpreted by physician consensus and in two ICU patient subgroups including those admitted for pneumonia and for rheumatologic/ endocrine diagnoses.
\end{abstract}

Results: Most reports were deemed 'negative' (51.8\%) by physician consensus. Many were 'possible' (41.7\%); only 6.5\% were 'positive' for pneumonia. The lexicon included 105 terms and uncertainty profiles that were encoded into 31 NLP queries. Queries identified 534,322 'hits' in the full sample, with $2.7 \pm 2.6$ 'hits' per report. An algorithm, comprised of twenty rules and probability steps, assigned interpretations to reports based on query profiles. In the validation set, the algorithm had $92.7 \%$ sensitivity, $91.1 \%$ specificity, $93.3 \%$ positive predictive value, and $90.3 \%$ negative predictive value for differentiating 'negative' from 'positive'/'possible' reports. In the ICU subgroups, the algorithm also demonstrated good performance, misclassifying few reports (5.8\%).

Conclusions: Many CXR reports in ICU patients demonstrate frank uncertainty regarding a pneumonia diagnosis. This electronic tool demonstrates promise for assigning automated interpretations to CXR reports by leveraging both terms and uncertainty profiles.

Keywords: Pneumonia, Intensive care unit, Natural language processing, Chest imaging, Electronic tool

\section{Background}

Pneumonia is a common cause of hospitalization [1,2]. In the intensive care unit (ICU), community- and hospitalacquired pneumonia are associated with substantial resource utilization, morbidity, and mortality [2,3]. Diagnosing pneumonia is often challenging since it requires both abnormal radiographic features and clinical findings $[1,4]$. In ICU patients, this diagnosis can be even more complex because of challenges in interpreting limited quality chest radiographs (CXRs) along with clinical data $[2,4,5]$.

\footnotetext{
* Correspondence: Vincent.X.Liu@kp.org

'Division of Research and Systems Research Initiative, Kaiser Permanente, 2000 Broadway, Webster Annex CA 94612 Oakland, Northern California ${ }^{2}$ Santa Clara Medical Center, Kaiser Permanente, Santa Clara, CA, Northern California

Full list of author information is available at the end of the article
}

Prior studies demonstrate the suitability of natural language processing (NLP) - a methodology for encoding data from narrative reports-for assisting with automated pneumonia identification within CXR reports [6-12]. While these techniques are promising, few studies have addressed the question of whether they perform accurately in the ICU [13]. Given the complexity of identifying pneumonia in ICU CXRs, little is known about the additional relevance of 'uncertainty' in the language used by interpreting radiologists [4].

In this study, we evaluate 194,615 CXR reports from patients in the ICU. In a manually reviewed sub-sample, we describe how pneumonia-related and uncertainty terms influence report interpretation. We then describe an electronic tool, comprised of NLP queries and an

\section{Biomed Central}


algorithm to evaluate query profiles, that assigns automated determinations ('positive,' possible', and 'negative') to reports. Finally, we evaluate its performance in a sample of reports drawn from ICU patient subgroups.

\section{Methods}

\section{Setting and participants}

The Kaiser Permanente Northern California (KPNC) Institutional Review Board approved this study. We conducted a retrospective analysis of CXR narrative reports from adult patients (age $\geq 18$ years) with ICU admissions at 21 KPNC hospitals between October 2007 and December 2010. All hospitals used the same electronic health information systems providing centralized access to clinical and radiographic data [14-18]. For study patients, we collected data from all CXR reports completed during a single hospitalization.

Our analysis included the development of (1) a pneumonia lexicon; (2) a set of NLP queries to identify lexicon terms within reports; and (3) an electronic algorithm that used query results to provide CXR report interpretation. The performance of these tools was measured in a validation set of CXR reports as well as in a set of reports from two patient subgroups.

\section{Lexicon development}

Two physicians experienced with critical care reviewed > 1,000 CXR reports to empirically develop a lexicon focused on categorizing features associated with pneumonia (Table 1) within three broad categories: (1) terms and term groups; (2) uncertainty profiles; and (3) 'other' features. Terms and term groups were broadly divided based on whether or not they would be seen in pneumonia. For example, pneumonia terms included those considered equivalent to pneumonia or likely to represent pneumonia (pneumonia-equivalent, e.g., bronchopneumonia or consolidation) as well as those used to convey a pneumonia diagnosis in the correct context (pneumonia-related, e.g., infiltrate or opacity). Non-pneumonia terms included those related to alternate processes (e.g., edema, atelectasis) or those conveying negative or unrelated findings ('no acute cardiopulmonary disease').

Uncertainty profiles were classified as having versus phrasing ('pneumonia versus atelectasis' or 'consolidation/ effusion'), low uncertainty ('probable pneumonia'), or high uncertainty ('cannot exclude infiltrate'; Table 1). Based on these elements, individual pneumonia terms (opacity) could be linked with uncertainty profiles (e.g., 'cannot exclude retrocardiac opacification'). The lexicon also encoded 'other' features relevant to interpreting radiograph reports including those assessing disease progression ('worsening of infiltrates'), anatomic location ('bilateral opacities'), or stability ('unchanged from prior').
Table 1 Development lexicon entries for terms and term groups and uncertainty profiles

\begin{tabular}{|c|c|c|c|}
\hline \multicolumn{2}{|c|}{ Terms and term groups } & \multicolumn{2}{|c|}{ Uncertainty profiles } \\
\hline Pneumonia-related & Non-pneumonia & $\begin{array}{l}\text { Low } \\
\text { uncertainty }\end{array}$ & $\begin{array}{l}\text { High uncertainty } \\
\text { or Versus }\end{array}$ \\
\hline Pneumonia & Atelectasis & Probable & Cannot exclude \\
\hline Bronchopneumonia & Edema & Consider & $\begin{array}{l}\text { Clinical } \\
\text { correlation }\end{array}$ \\
\hline Air bronchogram & $\begin{array}{l}\text { Congestive } \\
\text { heart failure }\end{array}$ & $\begin{array}{l}\text { Concerning } \\
\text { for }\end{array}$ & Could represent \\
\hline Consolidation & Heart failure & $\begin{array}{l}\text { Consistent } \\
\text { with }\end{array}$ & Possible \\
\hline Infiltrate & ARDS & Suspicious & Rule out \\
\hline Opacity & Fluid overload & Suspect & Questionable \\
\hline Density & Infarct & $\begin{array}{l}\text { Suggestive } \\
\text { of }\end{array}$ & Might \\
\hline Pneumonitis & Contusion & $\begin{array}{l}\text { Likely } \\
\text { representing }\end{array}$ & May \\
\hline Pneumonic & Hemorrhage & $\begin{array}{l}\text { Compatible } \\
\text { with }\end{array}$ & \\
\hline Abcess & Mass & & Versus \\
\hline Aspiration & $\begin{array}{l}\text { Low lung } \\
\text { volume }\end{array}$ & & Plus minus \\
\hline Cavity & Hypoinflation & & Or \\
\hline $\begin{array}{l}\text { Airspace disease/ } \\
\text { process }\end{array}$ & Congestion & & And/or \\
\hline \multirow{7}{*}{$\begin{array}{l}\text { Parenchymal } \\
\text { process }\end{array}$} & Malignancy & & / \\
\hline & Nodule & & \\
\hline & Neoplasm & & \\
\hline & Collapse & & \\
\hline & Effusion & & \\
\hline & Scar & & \\
\hline & Fluid & & \\
\hline
\end{tabular}

The table does not include all sub-combinations ('pneumonic infiltrate') or morphological variants ('clinical correlation' and 'clinically correlate').

\section{Natural language processing queries}

Based on this lexicon, we developed a set of query strategies to flag the presence of terms and phrases within CXR reports ('hits') using an NLP-based software package that enables semantic information extraction from large document collections (I2E, Linguamatics [www. linguamatics.com]; United Kingdom). We applied these queries to CXR reports using the I2E software to count the number of query hits within individual reports. Each query was designed to capture a combination of the terms, features, and uncertainty profiles defined by the lexicon. For example, a frequent uncertainty construct used by interpreting radiologists juxtaposes pneumonia with an alternate diagnosis (e.g.' 'pneumonia and/or atelectasis'). Thus, our corresponding query (termed 'pneumonia versus') would generate two hits for the phrases 
'atelectasis versus bronchopneumonia' and 'edema/pneumonia' within a single report. Queries were developed to incorporate focused negation so the phrases 'without evidence of edema and/or pneumonia' or 'no atelectasis/ pneumonia' would not generate hits, while the phrase 'no change in atelectasis versus pneumonia' would. Similar 'versus' queries were also designed to identify other pneumonia-related term groups (e.g., 'consolidation versus,' 'infiltrate + versus,' infection + versus').

\section{Physician interpretation}

To develop and validate our electronic algorithm for interpreting reports, we generated three sets of physicianinterpreted CXR reports (development, derivation, validation). For each report, two physicians experienced with interpreting ICU CXR reports reached a consensus on whether the report was 'positive,' 'possible', or 'negative' for pneumonia in a presumed scenario where CXRs were performed in patients whose clinical differential diagnosis included pneumonia (e.g., a patient with dyspnea). In the development $(n=777)$ and derivation $(n=950)$ sets, the physicians who created the lexicon and NLP queries assigned interpretations to randomly selected CXR reports. In the validation set, two other physicians (a radiologist and a pulmonary/critical care specialist) interpreted 739 additional CXR reports. The validation physicians had no role in the lexicon, query, and algorithm development; they were also blinded to the query and algorithm strategies.

\section{Electronic interpretation}

Using the gold-standard physician interpretations in the development and derivation sets, we then developed an electronic algorithm for assigning interpretations to CXR reports. The algorithm included twenty steps where each step incorporated rules- or probability-based strategies to analyze combinations of NLP query hits (Table 2). For example, a CXR report that included a 'blanket normal' statement (e.g., 'no acute cardiopulmonary findings') without any other pneumonia terms would be assigned a 'negative' interpretation. A report that included only pneumonia terms within high uncertainty profiles ('infiltrate versus atelectasis') would be assigned a 'possible' interpretation.

Because many reports included hits from several query elements that precluded simple rules-based interpretation, we also incorporated a set of predicted probabilities in selected algorithm steps. Using the development and derivation sets, we generated three logistic regression models to assign predicted probabilities that each report would have a 'positive', 'possible', or 'negative' interpretation. These probabilities were generated using backward stepwise logistic regression where NLP query hits associated with the binary outcome (e.g., for the 'negative only' outcome, negative $=1$ and positive or possible $=0$ ) with a $\mathrm{p}$ - value $<0.2$ were retained in the final model. The betacoefficients, based on the derivation sample, were then used to calculate probabilities in the validation sample (Additional file 1). These probabilities were then used in concert with NLP query profiles to assign interpretations to reports that could not be classified simply with rulesbased approaches. For example, after removing reports interpreted in the prior 11 steps, step 12 deemed a report 'negative' if its 'negative' predicted probability was $>30 \%$, its 'possible' probability was $<30 \%$, and its 'positive' probability was $<10 \%$.

\section{Algorithm performance}

We evaluated algorithm performance in the validation set based on sensitivity, specificity, positive predictive values, and negative predictive values. To collapse the outcome into binary values, these were calculated for 'Negative Alone' (where negative reports were distinguished from either positive or possible), 'Positive Alone' (positive reports versus negative or possible reports), and 'Possible Alone' (possible reports versus negative or positive reports) categories. We also evaluated cumulative test characteristics based on grouped algorithm steps to determine their impact on performance.

Finally, we evaluated the accuracy of the algorithm in two ICU subgroups expected to have a high percentage of either negative or positive/possible CXR reports-patients admitted with pneumonia $(\mathrm{n}=1,766)$ and with primarily rheumatologic or endocrine diagnoses $(n=1,201)$, as defined by Agency for Healthcare Research and Quality Clinical Classification Software codes (Additional file 1: Table S1) $[19,20]$. For both cohorts, we manually reviewed all 'unexpected' automated interpretation results (e.g., in the pneumonia cohort, a 'negative' CXR report within 48 hours of hospitalization would be an 'unexpected' finding) to assess whether the automated interpretations were accurate and categorize the report findings.

Analyses were conducted in Stata/SE 11.2 (College Station, TX). Results are reported as number (frequency) and mean \pm standard deviation.

\section{Results}

Study CXRs were randomly drawn from a total sample of 194,615 reports in 35,314 unique patients and 41,891 ICU admissions. Mean patient age was $65 \pm 17$ years; $52.6 \%$ of patients were male. Mean hospital length of stay was $8.8 \pm 13.8$ days. The mean number of CXR reports per patient was $4.2 \pm 6.4$.

\section{Physician interpretation}

Two physicians manually interpreted 2,466 CXR reports by consensus; Table 3 shows examples of reports and physician-based interpretations from the validation set. In general, reports suggestive of pneumonia but whose 
Table 2 Overview of electronic algorithm steps used to interpret chest radiograph reports based on rules- and probability-based strategies

\begin{tabular}{|c|c|c|c|}
\hline Group (Step) & Determination & Rules & Predicted probability \\
\hline Group 1 (Step 1) & Negative & $\begin{array}{l}\text { 'Blanket Negative' statement without any } \\
\text { pneumonia-related terms }\end{array}$ & \\
\hline Group 1 (Step 2) & Negative & No pneumonia-related terms & \\
\hline Group 1 (Step 3) & Possible & $\begin{array}{c}\text { High uncertainty pneumonia-related terms, } \\
\text { no 'blanket negative' statement }\end{array}$ & \\
\hline Group 1 (Step 4) & Positive & $\begin{array}{l}\text { Low/No uncertainty pneumonia-equivalent terms, } \\
\text { no high uncertainty pneumonia-related terms, } \\
\text { no non-pneumonia terms }\end{array}$ & \\
\hline Group 2 (Step 5) & Possible & $\begin{array}{l}\text { High uncertainty pneumonia-related terms, } \\
\text { no low/no uncertainty pneumonia-equivalent } \\
\text { terms, no normal statement }\end{array}$ & \\
\hline Group 2 (Step 6) & Possible & $\begin{array}{l}\text { Infiltrate + pneumonia-related terms, no low/ } \\
\text { no uncertainty pneumonia-equivalent terms }\end{array}$ & \\
\hline Group 2 (Step 7) & Possible & Any pneumonia-related versus terms & \\
\hline Group 3 (Step 8) & Positive & $\begin{array}{l}\text { Low/no uncertainty pneumonia-equivalent terms, } \\
\text { no blanket normal statement }\end{array}$ & \\
\hline Group 3 (Step 9) & Possible & Any uncertainty pneumonia-related terms & \\
\hline Group 3 (Step 10) & Possible & $\begin{array}{c}\text { Any infiltrate }+ \text { pneumonia-related terms, } \\
\text { no non-pneumonia terms }\end{array}$ & \\
\hline Group 4 (Step 11) & Positive & & Positive $>70 \%$ \\
\hline Group 4 (Step 12) & Negative & & Negative $>30 \%$, Possible $<30 \%$, Positive $<10 \%$ \\
\hline Group 4 (Step 13) & Possible & & Possible $>10 \%$, Negative $<10 \%$, Positive $<10 \%$ \\
\hline Group 4 (Step 14) & Possible & & Possible $>60 \%$, Negative $<40 \%$ \\
\hline Group 4 (Step 15) & Positive & Any pneumonia-equivalent term & \\
\hline Group 4 (Step 16) & Possible & & Possible $>20 \%$, Positive $>10 \%$ \\
\hline Group 4 (Step 17) & Possible & $\begin{array}{l}\text { Any uncertainty pneumonia-related terms, } \\
\text { no low/no uncertainty pneumonia-equivalent terms }\end{array}$ & Negative $<30 \%$ \\
\hline Group 4 (Step 18) & Possible & $\begin{array}{l}\text { Pneumonia-related terms, no non-pneumonia terms, } \\
\text { no blanket normal statement }\end{array}$ & Negative $<40 \%$ \\
\hline Group 4 (Step 19) & Negative & Non-pneumonia terms & \\
\hline Group 4 (Step 20) & Possible & All remaining reports & \\
\hline
\end{tabular}

Reports that are assigned an interpretation based on a step are then removed from interpretation in the subsequent steps.

findings could be seen in non-pneumonia conditions or required clinical data unavailable within the report were termed 'possible'. 'Negative' reports were not suggestive of pneumonia, however, they could be consistent with other conditions like congestive heart failure. Of all physician-reviewed reports, most were deemed 'negative' (Table 4; range, $47.0 \%$ to $57.4 \%$ ). A sizable fraction of reports were deemed 'possible' (overall, 41.7\%) while only a small fraction were felt to be conclusively 'positive' (overall, 6.5\%; validation, $7.2 \%$ ).

\section{Lexicon and query development}

The final lexicon included 52 terms/term groups, 27 uncertainty profiles, and 25 other terms/phrases not including morphological variants (e.g., infiltrate, infiltration, and infiltrative; (Table 1). In the final development stage, lexicon items, combinations, and uncer tainty profiles were encoded into 31 unique I2E NLP queries. Nine queries flagged high uncertainty pneumonia features (to identify phrases like infiltrate or edema,' 'pneumonia versus atelectasis'), nine flagged low uncertainty pneumonia features (e.g., 'probable pneumonia', 'suggestive of infiltrates'), five flagged nonpneumonia features (e.g., 'atelectasis', 'pleural effusion'), and eight flagged 'other' features (e.g., bilateral/multilobar location, new/progressive disease).

\section{I2E queries}

When applied to the total sample of 194,615 CXR reports, the $31 \mathrm{I} 2 \mathrm{E}$ queries produced a total of 534,322 hits. The mean number of hits per report was $2.7 \pm 2.6$, ranging from zero to 38. Additional file 1: Figure S1 shows a schematic example of the variety of query hits that would be identified in a CXR report interpreted as 'possible' pneumonia. In the 
Table 3 Selected examples of chest radiograph report determinations by category

\begin{tabular}{l} 
Positive \\
\hline $\mathbf{1}$ There is new bilateral lower lobe consolidation with air bronchograms. \\
There is some volume loss. Bibasilar pneumonias. \\
$\mathbf{2}$ Again noted is the focal consolidation at the right lung base. It is not \\
significantly changed and most likely represents middle lobe \\
pneumonia. Right middle lobe air space opacity is probably pneumonia \\
and not significantly changed. \\
Possible \\
$\mathbf{3}$ Interval clearing of the diffuse opacities of the lungs with residual \\
opacities, findings suggesting alveolar edema, less likely pneumonia. \\
$\mathbf{4}$ Endotracheal tube pulled back. Persistent cardiomegaly with congestive \\
heart failure and bilateral pleural effusions. Bibasilar pneumonia is \\
not excluded. \\
Negative \\
$\mathbf{5}$ Lungs are clear without pulmonary edema, focal consolidation, or \\
pleural effusion. No acute cardiopulmonary disease. \\
$\mathbf{6} \begin{array}{l}\text { Again seen are diffuse airspace opacities throughout both lungs, } \\
\text { improved compared with the most recent prior examination. The } \\
\text { pleural effusions appear smaller as well. Persistent pulmonary edema } \\
\text { though it appears improved. }\end{array}$
\end{tabular}

validation set, the queries identified a total of 2,228 hits, including 806 (36.2\%) for 'other', 638 (28.6\%) for non-pneumonia, 547 (24.6\%) for low uncertainty pneumonia, and 237 (10.6\%) for high uncertainty pneumonia features.

\section{Electronic algorithm}

The final electronic interpretation algorithm-based on testing in the development and derivation cohorts-was divided into 4 groups comprised of 20 steps (Table 2). The first 3 groups, including 10 steps, were entirely rulesbased; the 10 steps in the final group combined rules and predicted probabilities. For example, the first step in the algorithm encoded all CXR reports with a negative/normal phrase (e.g., 'no acute cardiopulmonary disease') and without any pneumonia-relevant terms as 'negative'. The third step encoded reports containing only low or no uncertainty pneumonia-equivalent phrases as 'positive'. Step 18, including both rules and probabilistic approaches, encoded reports as 'possible' if they included high uncertainty pneumonia-related terms and had a predicted

Table 4 Frequency of clinician interpretation for radiographs by sample

\begin{tabular}{lcccc}
\hline & \multicolumn{4}{c}{ Clinician interpretation, no. (\%) } \\
\hline Sample & $\boldsymbol{n}$ & Negative & Possible & Positive \\
Blinded validation & 739 & $424(57.4)$ & $262(35.5)$ & $53(7.2)$ \\
Derivation & 950 & $488(51.4)$ & $417(43.9)$ & $45(4.7)$ \\
Developmental & 777 & $365(47.0)$ & $350(45.0)$ & $62(8.0)$ \\
Overall & 2,466 & $1,277(51.8)$ & $1,029(41.7)$ & $160(6.5)$ \\
\hline
\end{tabular}

Table 5 Test characteristics of the automated interpretation algorithm by sample

\begin{tabular}{lcccc}
\hline & \multicolumn{4}{c}{ Test characteristics by interpretation samples (\%) } \\
\hline Dataset & Sensitivity & Specificity & PPV & NPV \\
& Negative-only (versus Positive or Possible) \\
Validation & 92.7 & 91.1 & 93.3 & 90.3 \\
Derivation & 93.2 & 96.8 & 96.8 & 93.1 \\
Overall & 92.8 & 93.1 & 93.5 & 92.3 \\
& Positive-only (versus Possible or Negative) \\
Validation & 45.3 & 99.0 & 77.4 & 95.9 \\
Derivation & 53.3 & 99.0 & 72.7 & 97.7 \\
Overall & 45.0 & 99.0 & 75.8 & 96.3 \\
& Possible-only (versus Positive or Negative) \\
Validation & 86.6 & 87.4 & 79.1 & 92.3 \\
Derivation & 94.2 & 89.9 & 87.9 & 95.2 \\
Overall & 89.9 & 87.5 & 83.8 & 92.4 \\
\hline PPV Positive & &
\end{tabular}

PPV Positive predictive value, NPV Negative predictive value.

probability of being negative of $<30 \%$. Table 5 shows the test characteristics of the algorithm in the derivation set.

\section{Validation set performance}

In the validation set, the performance of the algorithm was in a lower, but similar, range to that in the derivation set (Table 5). For the 'Negative Alone' category, the sensitivity was $92.7 \%$, specificity $91.1 \%$, positive predictive value 93.3\%, and negative predictive value 90.3\%. For the 'Positive Alone' category, the sensitivity (45.3\%) and positive predictive value (77.4\%) were substantially lower. For the 'Possible Alone' category, test characteristics ranged from 79.1\% (positive predictive value) to $92.3 \%$ (negative predictive value). Most CXR reports (70.2\%) could be categorized within the algorithm's first four steps (Additional file 1: Table S2). Those that could not be categorized by query rules alone-19.2\% of the total sample (group 4) were associated with worsened test characteristics.

\section{ICU sub-samples}

Among CXR reports in the ICU pneumonia cohort, the electronic algorithm interpreted 1,249 (70.7\%) as possible, $360(20.4 \%)$ as positive, and $157(8.9 \%)$ as negative. A manual review of the 157 unexpected 'negative' reports demonstrated that the algorithm misclassified seven reports $(4.5 \%$; Table 6$)$. The remaining reports were correctly interpreted and were either normal (31.8\%) or included radiologist interpretations consistent with nonpneumonia conditions (e.g., heart failure, 21.7\%). Among CXR reports for patients admitted with endocrine or rheumatologic diagnoses, the algorithm incorrectly interpreted $10(7.1 \%)$ reports. The remaining reports were suggestive of pneumonia or specifically communicated uncertainty about the diagnosis (Table 6). 


\begin{tabular}{|c|c|c|c|}
\hline \multicolumn{4}{|c|}{ Results by ICU admission diagnosis class } \\
\hline \multicolumn{2}{|l|}{ Pneumonia } & \multicolumn{2}{|c|}{ Endocrine/Rheumatologic } \\
\hline Category & Number (\%) & Category & Number (\%) \\
\hline Incorrect reading & $7(4.5)$ & Incorrect reading & $10(7.1)$ \\
\hline Normal report & $50(31.8)$ & $\begin{array}{l}\text { Pneumonia-relevant } \\
\text { term }\end{array}$ & $65(46.1)$ \\
\hline Heart failure & $34(21.7)$ & $\begin{array}{l}\text { Atelectasis versus } \\
\text { pneumonia-relevant }\end{array}$ & $40(28.4)$ \\
\hline $\begin{array}{l}\text { Other (e.g., mass, } \\
\text { nodules) }\end{array}$ & $27(17.2)$ & $\begin{array}{l}\text { Edema versus } \\
\text { pneumonia-relevant }\end{array}$ & $11(7.8)$ \\
\hline Atelectasis & $16(6.4)$ & Other & $8(5.7)$ \\
\hline Hypoinflation & $10(4.5)$ & Pneumonia & $7(5.0)$ \\
\hline $\begin{array}{l}\text { Interstitial } \\
\text { markings }\end{array}$ & $5(3.2)$ & & \\
\hline $\begin{array}{l}\text { Diaphragmatic } \\
\text { process }\end{array}$ & $4(2.5)$ & & \\
\hline $\begin{array}{l}\text { Scar/chronic } \\
\text { process }\end{array}$ & $4(2.5)$ & & \\
\hline
\end{tabular}

\section{Discussion}

In this study, we evaluated a large sample of chest radiograph reports from critically ill patients. Among nearly 2,500 reports categorized by manual review and physician consensus, $42 \%$ could not be classified as either 'negative' or 'positive'. In many cases, these 'possible' reports included language from interpreting radiologists that conveyed frank uncertainty about whether the findings represented pneumonia or another condition with an appearance similar to pneumonia. In these cases, interpreting physicians felt that additional clinical information, beyond the CXR report, were necessary to determine whether a pneumonia was present or absent. Only a minority of reports $(6.5 \%)$ included language that was deemed conclusive for, or highly likely to be, pneumonia.

In light of these challenges in categorizing ICU CXR reports into traditional 'negative' or 'positive' bins, we designed an algorithm that leveraged the wide range of uncertainty conveyed by radiologists. While this tool incorporated a set of complex techniques, the time required to analyze nearly 200,000 CXR reports-the estimated number of reports that would be generated at our 21 ICUs over 2 years-was as low as 10 minutes after document indexing. This electronic tool demonstrated very good performance in identifying 'negative' CXR reports. It also had high specificity for identifying 'positive' CXRs but had lower sensitivity and positive predictive value. Finally, it demonstrated good performance in identifying the sizable number of 'possible' CXR reports, a category that has not been well characterized in prior studies.

Pneumonia is a common and costly cause of hospitalization and is associated with substantial morbidity and mortality [1,2]. Among critically ill patients, hospitalacquired or ventilator-associated pneumonia further contribute to significant increases in length of stay, hospital costs, and mortality $[2,3]$. Prior studies have found that electronic tools can accurately identify abnormal radiograph reports and, thus, have the potential to improve clinical decision making and bedside care, quality and performance improvement, and adverse event or outcomes reporting [6-13,21-25]. Furthermore, when deployed on a large scale, these tools can be applied at a relatively low cost when compared with manual chart review. However, the interpretation tools in prior studies often considered CXR reports as a binary variable (negative/positive), limiting their diagnostic utility, especially in complex ICU patients [4].

A recent study by Dublin and others evaluated the performance of an open-source NLP system (ONYX) to assist with differentiating electronic CXR reports that required further manual review from those that could be conclusively labeled as 'consistent' or 'inconsistent' with pneumonia [26]. Out of 5,000 reports, between $12 \%$ and $25 \%$ were determined as requiring additional manual review-a lower, but still substantial, number of reports compared with our study. In their study, some criteria used to determine which reports required manual review were similar to those in our study (e.g., the presence of both atelectasis and pneumonia). In the remaining reports, their NLP system demonstrated excellent test characteristics similar to, or better than, those reported in prior NLP CXR report studies $[6,8,9,26,27]$. It is important to note the substantial differences in the patient populations from which the CXR reports were obtained. In the Dublin study, for example, $92 \%$ of reports were from outpatients-a population in whom radiographic image quality is expected to be higher and features like atelectasis or infiltrates are expected to be less prevalent [26].

Among inpatients, a new or progressive radiographic abnormality is necessary to raise the suspicion of pneumonia, however, the final diagnosis depends on a constellation of other clinical features (e.g., vital signs, symptoms, history, microbiology) [1,2]. In the ICU, diagnosing pneumonia is even more difficult because of technical challenges related to interpreting portable CXRs in supine patients with catheters, ventilators, devices, or competing conditions that can mimic pneumonia (e.g., fluid overload, atelectasis, lung hypo-inflation) $[4,5]$. Furthermore, in the ICU, the diagnosis of pneumonia can sometimes only be confirmed after treatment is administered and a patient's response is ascertained [2]. Our tool, which was built with these challenges in mind, helps extend the capabilities of prior NLP-based approaches that largely relied on a more proscribed set of terms without evaluating the significant uncertainty communicated by radiologists $[6,7,9,10,13]$.

Prior NLP studies have also evaluated the role of uncertainty in accurately interpreting biomedical reports [28-30]. 
For example, Vincze and others describe the development of the BioScope corpus which is annotated for a wide range of negations and linguistic speculations [28]. Many of the uncertainty profiles we captured in our lexicon are also described by the BioScope investigators including syntactic structures that connote ambiguity through auxiliaries, adjectives, or adverbs that are associated with keywords of interest. While the BioScope corpus contains free text from a wide variety of sources, including medical texts, biologic manuscripts, and abstracts, our corpus is drawn from a relatively proscribed source with a set of common and well-defined terms and phrases. As a result, the uncertainty profiles used in our NLP queries may have limited applicability to other free text sources. For example, common uncertainty phrases in CXR reports like cannot exclude infiltrates' may be infrequent in routine scholarly manuscripts or medical texts.

While our tool performed well independently, we designed it so that it could be overlaid with other detailed clinical, physiologic, and treatment data; essentially, the same data that clinicians use to confirm pneumonia in patients with an abnormal radiograph [2]. Using these additional diagnosis data in two ICU patient subgroups, we found that the algorithm continued to demonstrate very good performance in accurately assigning CXR report interpretations. We are currently incorporating this tool within more complex database structures that include detailed data about vital signs, ventilator settings and duration, antibiotic administration, and culture results [18]. This set of tools could be useful in a variety of healthcare domains. For example, in our healthcare system, quality improvement efforts aim to reduce the frequency of healthcare- or ventilatorassociated pneumonia, however, these efforts are limited by the resource strain of reviewing CXR reports among all hospitalized patients to identify relevant cases [2,31]. Our tool could be used to automatically evaluate all CXR reports in hospitalized patients and flag those whose cases require further detailed review. This tool could also be used in conjunction with electronic decision support tools that aid clinicians in correctly triaging pneumonia patients and choosing appropriate antibiotics $[11,25,31,32]$. Finally, as applied in the study by Dublin et al., these tools can aid in lowering the burden of chart review for research studies [26].

This study has several important limitations. First, while it included 21 hospitals, the CXR reports were all drawn from a single integrated healthcare delivery system in Northern California. It is possible that when applied to an external population of patients and interpreting radiologists, the performance of this algorithm might suffer because of differences in language across regions or institutions. Second, the queries were built within the proprietary I2E software package potentially presenting barriers to dissemination. However, we designed the query framework to be adaptable to other NLP-based search tools to foster future open-source availability. Finally, in this study, we developed these tools to analyze reports in a retrospective, rather than a real-time, setting. Our future development aims to provide real-time report indexing and querying to support the tool's applications at the point of bedside care.

\section{Conclusions}

More than 40 percent of chest radiograph reports from critically ill patients demonstrated uncertainty in assigning a diagnosis of pneumonia. An automated tool based on a set of natural-language processing-based queries and algorithms showed very good performance for accurately assigning 'positive,' 'possible', and 'negative' determinations in these reports, both when tested independently and in patient subgroups. This electronic tool demonstrates promise for using large-scale automated detection of suspicious findings from chest radiographs for clinical, operational, and reporting efforts.

\section{Additional file}

Additional file 1: Supplemental tables and figures for automated identification of pneumonia in chest radiograph reports in critically ill patients.

\section{Competing interests}

The authors declare that they have no competing interests.

\section{Authors' contributions}

$V L$ had full access to the data and takes responsibility for the integrity of the data and the accuracy of the data analysis. VL contributed to study design; data collection, analysis, and interpretation; preparation of the manuscript; and review and approval of the final manuscript. MC contributed to study design; data analysis and interpretation; preparation of the manuscript; and review and approval of the final manuscript. MM contributed to study design, data analysis, and preparation and approval of the final manuscript. RS contributed to study design, data analysis, and preparation and approval of the final manuscript. MG contributed to study design, data analysis and interpretation, and preparation and approval of the final manuscript. BT contributed to study design, data analysis, and preparation and approval of the final manuscript. GE contributed to study design, data analysis and interpretation, preparation of the manuscript, and review and approval of the final manuscript.

\section{Acknowledgments}

The authors thank the support staff from linguamatics for their assistance with I2E query design (Jeff Knauss, Jim Dixon, and Tracy Gregory) and Alina Schnake-Mahl for her assistance in manuscript formatting and preparation.

\section{Funding}

This work was supported by the Agency for Healthcare Quality and Research (AHRQ) grant 1R01HS018480-01, Novartis Vaccines and Diagnostics, Inc.,

The Permanente Medical Group, Inc., and Kaiser Foundation Hospitals, Inc. Dr. Vincent Liu was also supported in part by AHRQ grant F32HS019181-01.

\section{Prior abstract}

Portions of this research were presented at the 2012 and 2013 American Thoracic Society conferences. 


\section{Author details}

'Division of Research and Systems Research Initiative, Kaiser Permanente, 2000 Broadway, Webster Annex CA 94612 Oakland, Northern California. ${ }^{2}$ Santa Clara Medical Center, Kaiser Permanente, Santa Clara, CA, Northern California. ${ }^{3}$ Vallejo Medical Center, Kaiser Permanente, Vallejo, CA, Northern California. ${ }^{4}$ Walnut Creek Medical Center, Kaiser Permanente, Oakland, CA, Northern California.

Received: 20 March 2013 Accepted: 12 August 2013

Published: 15 August 2013

\section{References}

1. Mandell LA, Wunderink RG, Anzueto A, Bartlett JG, Campbell GD, Dean NC, Dowell SF, File TM Jr, Musher DM, Niederman MS, et al: Infectious diseases society of America/American thoracic society consensus guidelines on the management of community-acquired pneumonia in adults. Clinical infectious diseases: an official publication of the Infectious Diseases Society of America 2007, 44(Suppl 2):S27-S72.

2. Guidelines for the management of adults with hospital-acquired, ventilator-associated, and healthcare-associated pneumonia. American journal of respiratory and critical care medicine 2005, 17(4):388-416. PMID: 15699079

3. Chastre J, Fagon JY: Ventilator-associated pneumonia. American journal of respiratory and critical care medicine 2002, 165(7):867-903.

4. Henschke Cl, Yankelevitz DF, Wand A, Davis SD, Shiau M: Chest radiography in the ICU. Clinical imaging 1997, 21(2):90-103.

5. Ganapathy A, Adhikari NK, Spiegelman J, Scales DC: Routine chest x-rays in intensive care units: a systematic review and meta-analysis. Crit Care 2012, 16(2):R68.

6. Fiszman M, Chapman WW, Aronsky D, Evans RS, Haug PJ: Automatic detection of acute bacterial pneumonia from chest X-ray reports. Journal of the American Medical Informatics Association: JAMIA 2000, 7(6):593-604.

7. Hripcsak G, Austin JH, Alderson PO, Friedman C: Use of natural language processing to translate clinical information from a database of 889,921 chest radiographic reports. Radiology 2002, 224(1):157-163.

8. Mendonca EA, Haas J, Shagina L, Larson E, Friedman C: Extracting information on pneumonia in infants using natural language processing of radiology reports. Journal of biomedical informatics 2005, 38(4):314-321.

9. Asatryan A, Benoit S, Ma H, English R, Elkin P, Tokars J: Detection of pneumonia using free-text radiology reports in the BioSense system. International journal of medical informatics 2011, 80(1):67-73.

10. Bejan CA, Xia F, Vanderwende L, Wurfel MM, Yetisgen-Yildiz M: Pneumonia identification using statistical feature selection. Journal of the American Medical Informatics Association: JAMIA 2012, 19(5):817-823.

11. Vines C, Dean NC: Technology implementation impacting the outcomes of patients with CAP. Seminars in respiratory and critical care medicine 2012, 33(3):292-297.

12. Jones BE, Jones J, Bewick T, Lim WS, Aronsky D, Brown SM, Boersma WG van der Eerden MM, Dean NC: CURB-65 pneumonia severity assessment adapted for electronic decision support. Chest 2011, 140(1):156-163.

13. Haas JP, Mendonca EA, Ross B, Friedman C, Larson E: Use of computerized surveillance to detect nosocomial pneumonia in neonatal intensive care unit patients. American journal of infection control 2005, 33(8):439-443.

14. Selby JV: Linking automated databases for research in managed care settings. Annals of internal medicine 1997, 127(8 Pt 2):719-724.

15. Go AS, Hylek EM, Chang Y, Phillips KA, Henault LE, Capra AM, Jensvold NG, Selby JV, Singer DE: Anticoagulation therapy for stroke prevention in atrial fibrillation: how well do randomized trials translate into clinical practice? JAMA: the journal of the American Medical Association 2003, 290(20):2685-2692.

16. Escobar GJ, Greene JD, Scheirer P, Gardner MN, Draper D, Kipnis P: Risk-adjusting hospital inpatient mortality using automated inpatient, outpatient, and laboratory databases. Medical care 2008, 46(3):232-239.

17. Escobar GJ, Fireman BH, Palen TE, Gardner MN, Lee JY, Clark MP, Kipnis P. Risk adjusting community-acquired pneumonia hospital outcomes using automated databases. The American journal of managed care 2008, 14(3):158-166.

18. Escobar GJ, LaGuardia JC, Turk BJ, Ragins A, Kipnis P, Draper D: Early detection of impending physiologic deterioration among patients who are not in intensive care: development of predictive models using data from an automated electronic medical record. Journal of hospital medicine: an official publication of the Society of Hospital Medicine 2012, 7(5):388-395.

19. Cowen ME, Dusseau DJ, Toth BG, Guisinger C, Zodet MW, Shyr Y: Casemix adjustment of managed care claims data using the clinical classification for health policy research method. Medical care 1998, 36(7):1108-1113.

20. Healthcare Cost and Utilization Project (HCUP): Agency for healthcare research and quality; 2013. www.hcup-us.ahrq.gov/toolssoftware/ccs/ccs.jsp.

21. Hripcsak G, Friedman C, Alderson PO, DuMouchel W, Johnson SB, Clayton PD: Unlocking clinical data from narrative reports: a study of natural language processing. Annals of internal medicine 1995, 122(9):681-688.

22. Meystre SM, Savova GK, Kipper-Schuler KC, Hurdle JF: Extracting information from textual documents in the electronic health record: a review of recent research. Yearbook of medical informatics 2008, PMID:18660887:128-144

23. Azzam HC, Khalsa SS, Urbani R, Shah CV, Christie JD, Lanken PN, Fuchs BD: Validation study of an automated electronic acute lung injury screening tool. Journal of the American Medical Informatics Association: JAMIA 2009, 16(4):503-508.

24. Demner-Fushman D, Chapman WW, McDonald CJ: What can natural language processing do for clinical decision support? Journal of biomedical informatics 2009, 42(5):760-772.

25. Sevenster M, van Ommering R, Qian Y: Bridging the text-image gap: a decision support tool for real-time PACS browsing. Journal of digital imaging: the official journal of the Society for Computer Applications in Radiology 2012, 25(2):227-239.

26. Dublin $\mathrm{S}$, Baldwin E, Walker RL, Christensen LM, Haug PJ, Jackson ML, Nelson JC, Ferraro J, Carrell D, Chapman WW: Natural Language Processing to identify pneumonia from radiology reports. Pharmacoepidemiology and drug safety 2013, 22(8):834-841.

27. Elkin PL, Froehling D, Wahner-Roedler D, Trusko B, Welsh G, Ma H, Asatryan AX, Tokars Jl, Rosenbloom ST, Brown SH: NLP-based identification of pneumonia cases from free-text radiological reports. AMIA Annual Symposium proceedings/ AMIA Symposium AMIA Symposium 2008, 18998791:172-176.

28. Vincze V, Szarvas G, Farkas R, Mora G, Csirik J: The BioScope corpus: biomedical texts annotated for uncertainty, negation and their scopes. BMC bioinformatics 2008(9):11-S9.

29. Agarwal $\mathrm{S}, \mathrm{Yu} \mathrm{H}$ : Detecting hedge cues and their scope in biomedical text with conditional random fields. Journal of biomedical informatics 2010, 43(6):953-961

30. Agarwal $\mathrm{S}, \mathrm{Yu} \mathrm{H}$ : Biomedical negation scope detection with conditional random fields. Journal of the American Medical Informatics Association: JAMIA 2010, 17(6):696-701.

31. Gellad WF, Yealy D, Fine M: Computers and the diagnosis of pneumonia: comment on "performance and utilization of an emergency department electronic screening tool for pneumonia". JAMA internal medicine 2013, 173(8):701-702.

32. Dean NC, Jones BE, Ferraro JP, Vines CG, Haug PJ: Performance and utilization of an emergency department electronic screening tool for pneumonia. JAMA internal medicine 2013, 173(8):699-701.

doi:10.1186/1472-6947-13-90

Cite this article as: Liu et al:: Automated identification of pneumonia in chest radiograph reports in critically ill patients. BMC Medical Informatics and Decision Making 2013 13:90.

\section{Submit your next manuscript to BioMed Central and take full advantage of:}

- Convenient online submission

- Thorough peer review

- No space constraints or color figure charges

- Immediate publication on acceptance

- Inclusion in PubMed, CAS, Scopus and Google Scholar

- Research which is freely available for redistribution 